\title{
Bifurcations Associated with Sub-Synchronous Resonance
}

\author{
Yasunori Mitani and Kiichiro Tsuji \\ Osaka University, Suita, Osaka 565, Japan \\ Matthew Varghese*, Felix F. Wu and Pravin Varaiya \\ *Landis \& Gyr Utilities Services Inc., San Jose, CA 95110 \\ University of California, Berkeley, CA 94720
}

\begin{abstract}
This paper describes a set of results of detecting nonlinear phenomena appearing in a turbine generator power system with series-capacitor compensation. The analysis was based on the Floquet theory as well as the Hopf Bifurcation theorem. After the first Hopf bifurcation, the stable limit cycle bifurcates to a stable torus and an unstable limit cycle which connects to a stable limit cycle by a supercritical torus bifurcation. The stable limit cycle joins with an unstable limit cycle at a cyclic fold bifurcation. This unstable limit cycle is connected to the second Hopf. It has been also numerically demonstrated that such a strange sequence of periodic orbits is created by a q-axis damper winding.
\end{abstract}

Keywords-Bifurcation, Floquet multiplier, Nonlinear analysis, Subsynchronous resonance, Series capacitor compensation, Power system dynamics

\section{INTRODUCTION}

We study the phenomenon of SSR (Sub-Synchronous Resonance) in models of turbine-generator systems with series-capacitor compensation. The usual analysis of SSR is based on frequency domain methods using linear models such as in [2]. Some controllers using electronically switched transmission line compensators, which are collectively known as FACTS (Flexible Alternating Current Transmission Systems), have been designed also using frequency domain analysis and proven to be effective in mitigating the deleterious effects of SSR in [4] and [5]. However, studies in [6] and [7] indicate that because of the stable limit cycle with small magnitude existing after the Hopf bifurcation, the range of instability of torsional oscillations is narrower than the one which is predicted by an eigenanalysis method.

PE-337-PWRS-6-02-1997 A paper recommended and approved by the IEEE Power System Engineering Committee of the IEEE Power Engineering Society for publication in the IEEE Transactions on Power Systems Manuscript submitted December 22, 1995; made available for printing February 20, 1997.
This paper presents the analysis of limit cycle based on the Floquet theory as well as the Hopf bifurcation theorem. Section 2 contains some mathematical preliminaries. The 12 th order turbine-generator model is described in Section 3. Results of numerical studies using AUTO94 [8] are given in Section 4. It should be noted that the torus bifurcation, documented in Section 4, has not been reported in SSR studies such as [4], [5], [6] or [7]. The result shows a surprising similarity to the 2 machine system with nonlinear dynamics [18].

\section{Mathematical Preliminaries}

\section{A. Supercritical and Subcritical Hopf}

The following discussion is adapted from [9], [10], [15], [17]. Any parameterized two-dimensional system with an equilibrium at 0 and purely imaginary eigenvalues, can be cast by a smooth change of coordinates into the polar form

$$
\begin{aligned}
& \dot{r}=\left(d \gamma+a r^{2}\right) r \\
& \dot{\theta}=\omega+c \gamma+b r^{2} .
\end{aligned}
$$

In general, the equilibrium need not be at the origin. If $d \neq 0$, there is a bifurcation at $\gamma=0$ since the origin is stable for $d \gamma<0$ and unstable for $d \gamma>0$. Since the first equation does not depend on $\theta$, we see immediately that there are periodic orbits, $r=$ constant $\neq 0$. If $a \neq 0$ and $d \neq 0$ these solutions lie along the parabola $d \gamma+a r^{2}=$ 0 . The Hopf bifurcation theorem says that this situation holds in general. The following hypotheses are needed:

1. The system $\dot{x}=f(x, \gamma)$ has an isolated equilibrium at $x_{0}(\gamma)$.

2. $f(x, \gamma)$ is $C^{r}(r \geq 4)$.

3. The Jacobian $\partial f\left(x_{0}(\gamma), \gamma\right) / \partial x$ possesses a pair of complex conjugate, simple eigenvalues

$$
\lambda(\gamma)=\alpha(\gamma)+j \omega(\gamma), \quad \bar{\lambda}(\gamma)=\alpha(\gamma)-j \omega(\gamma)
$$

4. At the critical value

$$
\begin{aligned}
& \quad \alpha\left(\gamma_{0}\right)=0, \omega_{0}:=\omega\left(\gamma_{0}\right)>0, \quad \text { and } \alpha^{\prime}\left(\gamma_{0}\right)>0 \\
& \text { Besides } \pm j \omega_{0}, \text { the other eigenvalues of the critical } \\
& \text { Jacobian } \partial f\left(x\left(\gamma_{0}\right), \gamma_{0}\right) / \partial x \text { have strictly negative real } \\
& \text { part. }
\end{aligned}
$$


The following existence and uniqueness conditions are from the Hopf bifurcation theorem in [15].

Existence. There is $\epsilon_{H}>0$ and a $C^{r-1}$ function

$$
\gamma(\epsilon)=\gamma_{0}+\gamma_{2} \epsilon^{2}+O\left(\epsilon^{3}\right)
$$

and for each $0<\epsilon<\epsilon_{H}$ there is a nonconstant periodic solution $x_{\epsilon}(t)$ near the equilibrium $x_{0}(\gamma)$ for the parameter value $\gamma=\gamma(\epsilon)$. The period of $x_{\epsilon}$ is a $C^{r-1}$ function

$$
T(\epsilon)=2 \pi \omega_{0}^{-1}\left[1+T_{2} \epsilon^{2}\right]+O\left(\epsilon^{3}\right)
$$

and its amplitude grows as $O(\epsilon)$.

Uniqueness. If $\gamma_{2} \neq 0$ then there is $\epsilon_{0}>0$ such that for each $0<\epsilon<\epsilon_{0}, x_{\epsilon}$ is the only periodic orbit for $\gamma=\gamma(\epsilon)$ near $x_{0}(\gamma(\epsilon))$.

Stability. Exactly one of the characteristic exponents of $x_{\epsilon}(t)$ approaches 0 as $\epsilon \rightarrow 0$, and it is given by the real $C^{r-1}$ function

$$
\beta(\epsilon)=\beta_{2} \epsilon^{2}+O\left(\epsilon^{3}\right)
$$

The relationship

$$
\beta_{2}=-2 \alpha^{\prime}\left(\gamma_{0}\right) \gamma_{2}
$$

holds. Moreover the periodic solution $x_{\epsilon}(t)$ is orbitally asymptotically stable with asymptotic phase if $\beta(\epsilon)<0$ but is unstable if $\beta(\epsilon)>0$. The bifurcation is said to be supercritical in the former case and subcritical in the latter. For the two dimensional case, the formula for $\beta_{2}$ is given by

$$
\begin{aligned}
8 \beta_{2} & =\frac{1}{\omega_{0}}\left\{f_{11}^{1}\left(f_{11}^{2}-f_{12}^{1}\right)+f_{22}^{2}\left(f_{12}^{2}-f_{22}^{1}\right)\right. \\
& \left.+\left(f_{11}^{2} f_{12}^{2}-f_{12}^{1} f_{22}^{1}\right)\right\} \\
& +\left(f_{111}^{1}+f_{122}^{1}+f_{112}^{1}+f_{222}^{2}\right)
\end{aligned}
$$

Here

$$
\begin{gathered}
f=\left(f^{1}, f^{2}\right), \\
f_{p q}^{i}=\frac{\partial^{2} f^{i}}{\partial x_{p} \partial x_{q}}\left(0, \gamma_{0}\right), \\
f_{p q r}^{i}=\frac{\partial^{3} f^{i}}{\partial x_{p} \partial x_{q} \partial x_{r}}\left(0, \gamma_{0}\right)
\end{gathered}
$$

In the general case, one uses the center manifold theorem and perturbation analysis with multiple time scales to reduce an $n$ dimensional system satisfying the hypotheses to a two-dimensional model.

\section{B. Cyclic Fold and Torus Bifurcations}

Consider a differential equation $\dot{x}=f(x), x \in \Re^{n}$. Denote its solution starting at a point $x$ by $\phi_{t}(x)=\phi(t, x)$. The map $\phi:(t, x) \rightarrow \phi(t, x)$ is called the flow of the vector field $f(x)$. Let $\Gamma$ be a periodic orbit of period $T$ of the flow $\phi$. We first take a local cross section $S \subset \Re^{n}$, of dimensional $n-1$, such that (i) the flow $\phi$ is everywhere transverse to $S$ (This means the inner product $\langle f(x), n(x)>\neq 0$ for all $x \in S$, where $n(x)$ is the unit normal to $S$ at $x$.), and (ii) $S$ intersects $\Gamma$ at a unique point $p$. Let $\mathcal{U} \subset S$ be a neighbourhood of $p$. For $q \in \mathcal{U}$ the first return or Poincaré map $\mathcal{P}: \mathcal{U} \rightarrow S$ is defined by

$$
\mathcal{P}(q):=\phi_{\tau(q)}(q)=\phi(\tau(q), q)
$$

where $\tau(q)$ is the time it takes for the orbit based at $q$ to first return to $S$. Note that $\tau(p)=T$ and $\tau(q) \rightarrow T$ as $q \rightarrow p$. Also $p$ is a fixed point of $\mathcal{P}$. In local coordinates, $\mathcal{P}$ is a map from $\mathcal{U} \subset \Re^{n-1}$ to $\Re^{n-1}$, so $D \mathcal{P}(p)$ has $n-1$ eigenvalues. The eigenvalues of $D \mathcal{P}(p)$ are the same as $n-1$ eigenvalues of $D \phi_{T}(p)$, and are called the Floquet multipliers (or characteristic multipliers) associated with the periodic orbit $\Gamma$.

Suppose the system has a periodic orbit for a parameter value $\mu$. Denote the Floquet multipliers by $\lambda_{1}(\mu), \cdots, \lambda_{n-1}(\mu)$. If the periodic solution is stable, then $\lambda_{1}(\mu), \cdots, \lambda_{n-1}(\mu)$ are all inside the unit circle. The multipliers are functions of the parameter $\mu$. As $\mu$ is varied, some of them may cross the unit circle at a critical value $\mu_{0}$. A multiplier crossing the unit circle is called a critical multiplier. Several types of branching may occur depending on where a multiplier or a pair of complex conjugate multipliers exits the unit circle. There are three possible cases. In the numerical study, we only observed Case 1 and Case 3.

1. Cyclic Fold: Only one multiplier crosses the unit circle along the positive real axis with $\lambda_{i}\left(\mu_{0}\right)=1$ for some $1 \leq i \leq n-1$.

2. Period Doubling: Only one multiplier crosses the unit circle along the negative real axis with $\lambda_{i}\left(\mu_{0}\right)=$ -1 for some $1 \leq i \leq n-1$.

3. Torus: Only one pair of complex conjugate multipliers crosses the unit circle with $\left|\lambda_{i}\left(\mu_{0}\right)\right|=1$, $\operatorname{Im}\left(\lambda_{i}\left(\mu_{0}\right)\right)=-\operatorname{Im}\left(\overline{\lambda_{i}}\left(\mu_{0}\right)\right)>0$, and $\frac{d}{d \mu}\left|\lambda_{i}\left(\mu_{0}\right)\right| \neq 0$ for some $1 \leq i \leq n-1$.

\section{The 12Th order Turbine-Generator Model}

\section{A. System Description}

The configuration of the single-machine to infinite bus power system used in this study is shown in Fig.1.

The notation used here is standard [1], [2], [3] and will not be elaborated further; $\omega_{0}$ is the frequency of the voltage at the infinite bus, $d$ and $q$ refer to the direct and quadrature axes. The 12 variables are armature winding flux $\left(\Phi_{d}, \Phi_{q}\right)$, field winding flux $\left(\Psi_{f}\right)$, damper winding flux $\left(\Psi_{k d}, \Psi_{k q 1}, \Psi_{k q 2}\right)$, absolute angle of the generator $\left(\theta_{1}\right)$, relative angle of turbine $\left(\lambda_{2}\right)$, angular velocities of 


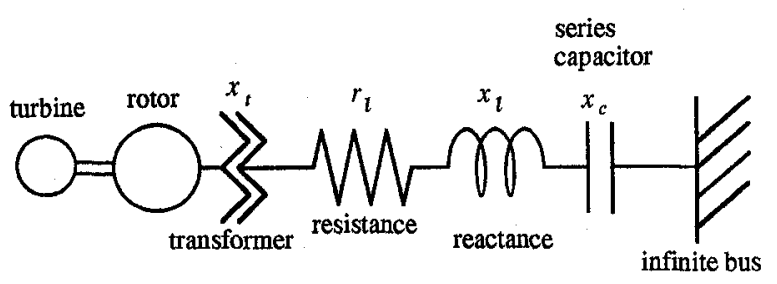

Fig. 1. Configuration of power system for study.

the generator and turbine $\left(\omega_{1}, \omega_{2}\right)$ and voltages of the series capacitor $\left(V_{c d}, V_{c q}\right)$, where $\lambda_{2}=\theta_{2}-\theta_{1}$ and the absolute angle of the turbine is $\theta_{2}$. Note that, the 12th order model has only 1 torsional mode, no AVR and no governor. This model is the simplest system that can exhibit subsynchronous resonance.

$$
\begin{aligned}
\dot{\theta_{1}} & =\omega_{1}-1 \\
\dot{\lambda_{2}} & =\omega_{2}-\omega_{1} \\
M_{1} \omega_{0} \dot{\omega_{1}} & =-D_{11}\left(\omega_{1}-1\right)-D_{12}\left(\omega_{1}-\omega_{2}\right) \\
& +K_{12} \lambda_{2}-\left(\Phi_{d} i_{q}-\Phi_{q} i_{d}\right) \\
M_{2} \omega_{0} \dot{\omega_{2}} & =-D_{22}\left(\omega_{2}-1\right)-D_{12}\left(\omega_{2}-\omega_{1}\right) \\
& -K_{12} \lambda_{2}+T_{m 2} \\
\dot{\Phi_{d}} & =+\omega_{1} \Phi_{q}+R_{A} i_{d}+V_{c d}+E_{s} \sin \left(\theta_{1}\right) \\
\dot{\Phi_{q}} & =-\omega_{1} \Phi_{d}+R_{A} i_{q}+V_{c q}+E_{s} \cos \left(\theta_{1}\right) \\
\dot{\Psi_{f}} & =-R_{F} i_{f}+E_{F D} \\
\dot{\Psi_{k d}} & =-R_{k d} i_{k d} \\
\dot{\Psi}_{k q 1} & =-R_{k q 1} i_{k q 1} \\
\dot{\Psi}_{k q 2} & =-R_{k q 2} i_{k q 2} \\
\dot{V_{c d}} & =+\omega_{1} V_{c q}+X_{c} i_{d} \\
\dot{V_{c q}} & =-\omega_{1} V_{c d}+X_{c} i_{q}
\end{aligned}
$$

$\Phi_{d}=\Psi_{d}-x_{d} i_{d}$ and $\Phi_{q}=\Psi_{q}-x_{q} i_{q} . i_{d}, i_{f}$ and $i_{k d}$ are linear combinations of $\Phi_{d}, \Psi_{f}$ and $\Psi_{k d} . i_{q}, i_{k q 1}$ and $i_{k q 2}$ are linear combinations of $\Phi_{q}, \Psi_{k q 1}$ and $\Psi_{k q 2}, \tau$ is the time variable and $\tau=\omega_{0} t$ is the time scale. $X_{c}$ is the negative reactance due to series-capacitor compensation.

\section{B. System Constants}

Two sets of system constants are provided; (A) A model with a long distance power transmission line, where it has only one damper winding in q-axis. (B) A model cited from the IEEE first benchmark model [19], [3] except that it has only one turbine, no AVR and no governor. System constants are shown in Table 1.

\section{Nomenclature for Eigenanalysis}

(A) LC modes are associated with eigenvalues due to $\Phi_{d}, \Phi_{q}, V_{c d}, V_{c q}$ dynamics. The name "LC" is derived from
TABLE I

SYSTEM CONSTANTS

\begin{tabular}{llll}
\hline model A & & & \\
$x_{a d}=1.350$ & $x_{d}=1.600$ & $x_{f}=1.440$ & $x_{k d}=1.380$ \\
$x_{a q}=1.350$ & $x_{q}=1.600$ & $x_{k q}=1.370$ & \\
$r_{f}=0.0006$ & $r_{k d}=0.0062$ & $r_{k q}=0.0124$ & \\
$r_{a}=0.0018$ & & & \\
$M_{1}=5.57$ & $M_{2}=2.21$ & $D_{11}=0.5$ & $D_{22}=0.5$ \\
$D_{12}=1.2$ & $K_{12}=25.0$ & & \\
$x_{t}=0.197$ & $x_{l}=1.593$ & $r_{l}=0.04626$ & \\
\hline model B & & & \\
$x_{a d}=1.660$ & $x_{d}=1.790$ & $x_{f}=1.700$ & $x_{k d}=1.666$ \\
$x_{a q}=1.580$ & $x_{q}=1.710$ & $x_{k q 1}=1.695$ & $x_{k q 2}=1.825$ \\
$r_{f}=0.001$ & $r_{k d}=0.0037$ & $r_{k q 1}=0.0053$ & $r_{k q 2}=0.0182$ \\
$r_{a}=0.0015$ & & & \\
$M_{1}=3.9827$ & $M_{2}=1.3054$ & $D_{11}=0.5$ & $D_{22}=0.5$ \\
$D_{12}=0.6$ & $K_{12}=150.0$ & & \\
$x_{t}=0.14$ & $x_{l}=0.56$ & $r_{l}=0.0315$ & \\
\hline
\end{tabular}

the 'inductive' nature of the $\Phi_{d}, \Phi_{q}$ dynamics and the 'capacitive' nature of the $V_{c d}, V_{c q}$ dynamics. The 4 eigenvalues form 2 complex conjugate pairs that coincide at $X_{c}=0$. As $X_{c}$ is increased the eigenvalues separate. The eigenvalues that move away from the real axis are called super-synchronous and the pair that moves toward the real axis are called sub-synchronous. For values of $X_{c}$ close to zero, the LC modes have a frequency of about $360 \mathrm{rad} / \mathrm{sec}$. Of primary interest is the movement of the sub-synchronous eigenvalues since these interact with the eigenvalues of the torsional modes. Little work has been done on the super-synchronous eigenvalues but it is important to note that if these eigenvalues move to a very high $\omega$ value, then there could be possible interaction with the high frequency behavior of thyristor switches. Some examples of such harmonic induced switching time bifurcations can be found in [16].

(B) Torsional mode eigenvalues arise out of the variables $\lambda_{2}, \omega_{2}$ associated with turbine dynamics. The torsional modes have a frequency of about $100 \mathrm{rad} / \mathrm{sec}$.

(C) EM (Electro-Mechanical) modes are associated with the variables $\theta_{1}, \omega_{1}$ associated with generator dynamics. The frequency is about $6 \mathrm{rad} / \mathrm{sec}$.

(D) Damper windings eigenvalues are associated with the $\Psi_{k d}, \Psi_{k q 1}, \Psi_{k q 2}$ variables. These eigenvalues have no imaginary components in this case.

(E) Field winding eigenvalue, from variable $\Psi_{f}$, has no imaginary component since there is no AVR. The $\Psi_{f}$ eigenvalue is about $10^{-2}$ smaller than the damper eigenvalues.

\section{Nonlinear ANalysis of SSR by AUTO94}

\section{A. Interaction between $L C$ and torsional modes}

The interaction of the subsynchronous $L C$ mode and the torsional mode leads to 3 Hopf bifurcations and Fig.2 shows the downward movement of the $L C$ eigenvalue as 


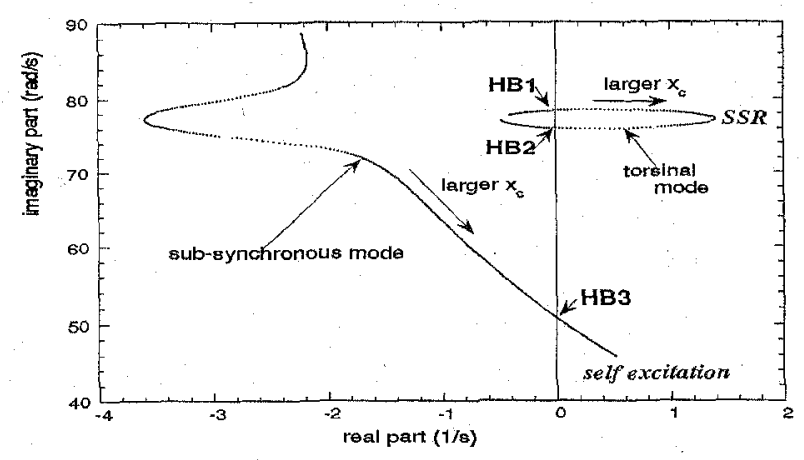

Fig. 2. behavior of eigenvalues w.r.t. $X_{c}$ (model A).

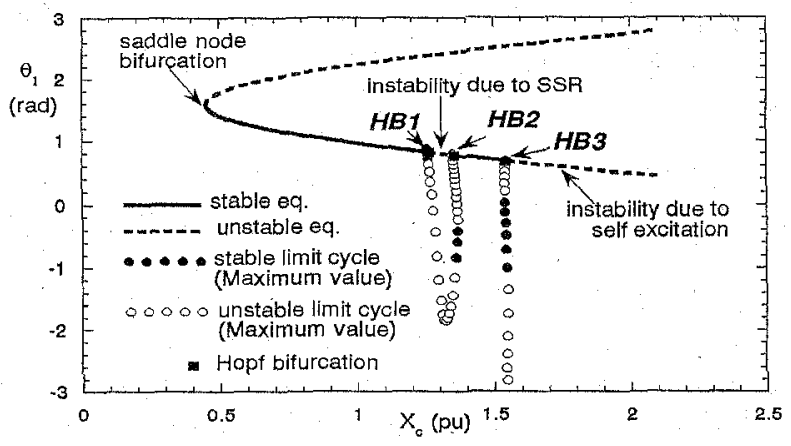

Fig. 3. Bifurcation diagram (model A).

$X_{c}$ is increased in model A. When the $L C$ eigenvalue nears the $\omega$ location of the torsional eigenvalue, the latter moves to the right. Eventually, the torsional eigenvalue moves across the $j \omega$ axis. This signifies a Hopf bifurcation. In Fig.2, this is labeled $H B 1$. As the $L C$ moves further downward, it causes the torsional eigenvalue to return to the left half plane. This is the second Hopf bifurcation. In Fig.2, this is labeled $H B 2$. After $H B 2$ the torsional dynamics remain stable. However, on further increase of $X_{c}$, the $L C$ eigenvalue crosses the $j \omega$ axis to the right half plane and the system has no stable equilibrium points. In Fig.2, this is labeled $H B 3$.

\section{B. The Other Modes: EM, Damper and Field}

The plot in Fig.2 does not show the EM, damper and field winding eigenvalues because these remain in the left half plane. Compare these results to the Hopf bifurcation case studies, where it is the $E M$ mode that is the primary cause of nonlinear oscillations [11], [12], [13], [14].

\section{The Main Bifurcation Diagram}

The main bifurcation diagrams are shown in Fig.3 for model A and in Fig.4 for model B. It is observed that both figures are qualitatively same. Fig. 5 is a magnification of the periodic orbit in Fig. 3 detailing the sequence of supercritical Hopf, torus bifurcations, cyclic-fold and

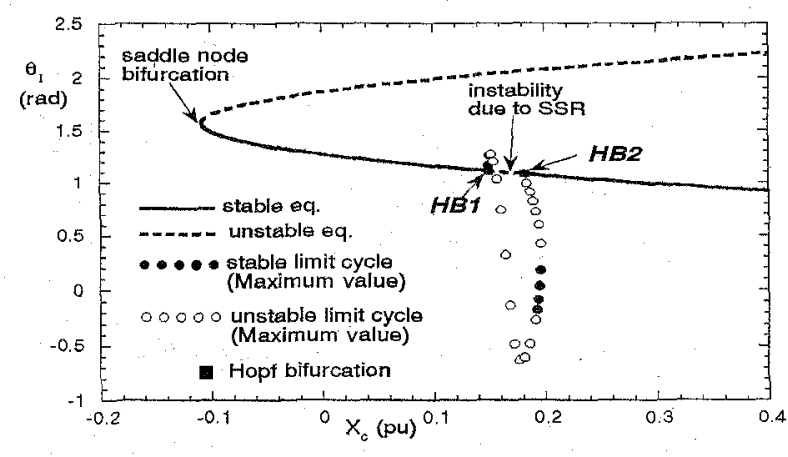

Fig. 4. Bifurcation diagram (model B).

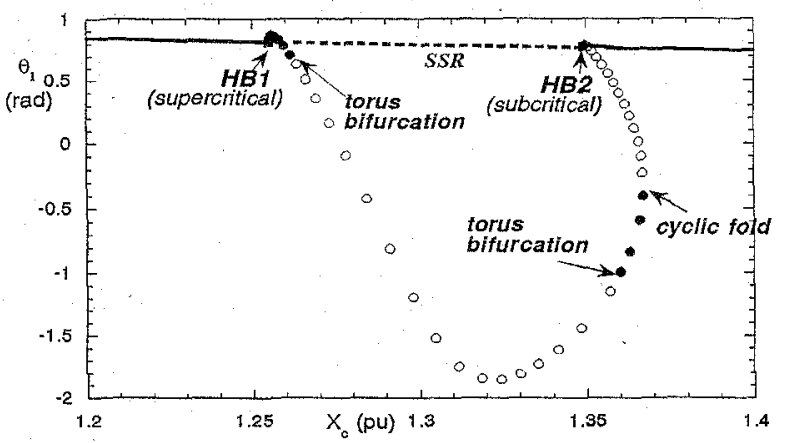

Fig. 5. Magnification of the periodic orbit (model A).

subcritical Hopf bifurcations. Fig.5 shows a surprising similarity to the 4 variable ( 2 machine) system with load dynamics [18]. The following comparisons can be drawn:

(I) The similarity is that the stable equilibrium branch undergoes an approximately identical sequence of bifurcations namely: Subcritical Hopf $\rightarrow$ Cyclic Fold $\rightarrow$ Stable Limit Cycle $\rightarrow$ Attractor $\rightarrow$ No Attractors $\rightarrow$ Attractor $\rightarrow$ Stable Limit Cycle $\rightarrow$ Supercritical Hopf.

(II) One difference is that the bifurcations for the 2 machine system are load-induced, whereas the bifurcations for SSR occur by the interaction between $L C$ and torsional eigenvalues.

(III) Another difference is that in the 2 machine system the attractor was chaotic, whereas the attractor is a stable torus in the SSR system.'

(IV) Whereas, the destruction of the chaotic attractor of the 2 machine system via a boundary crisis has been proved, it is still not clear how the window of no stable attractors for the SSR system is establishod.

(V) Finally, the region of instability, beyond the Hopf bifurcation point labeled $H B 3$ in Fig.2 does not exist for the 2 machine system.

\section{Detail of Periodic Orbits}

Fig.6 shows the supercritical Hopf in $\theta_{1}-\omega_{1}$ space for $X_{c}=1.260$ of model A. After the supercritical Hopf 


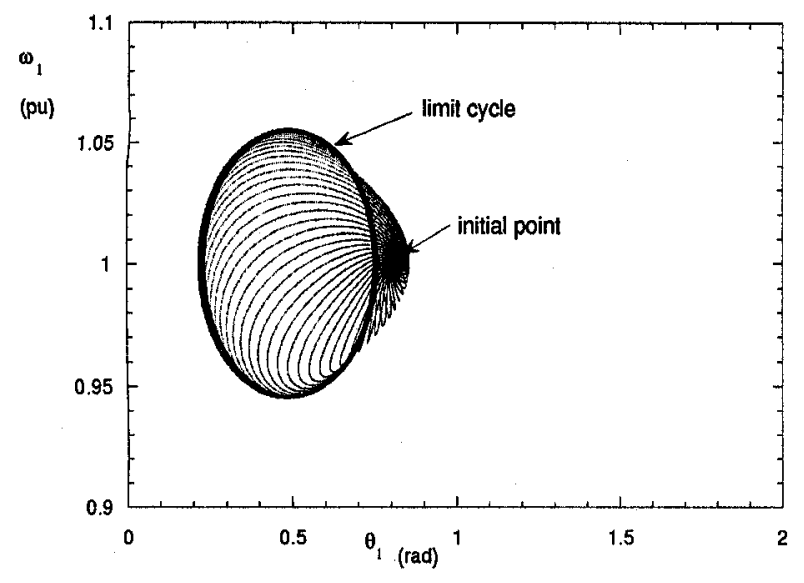

Fig. 6. Limit cycle at $X_{c}=1.260$.

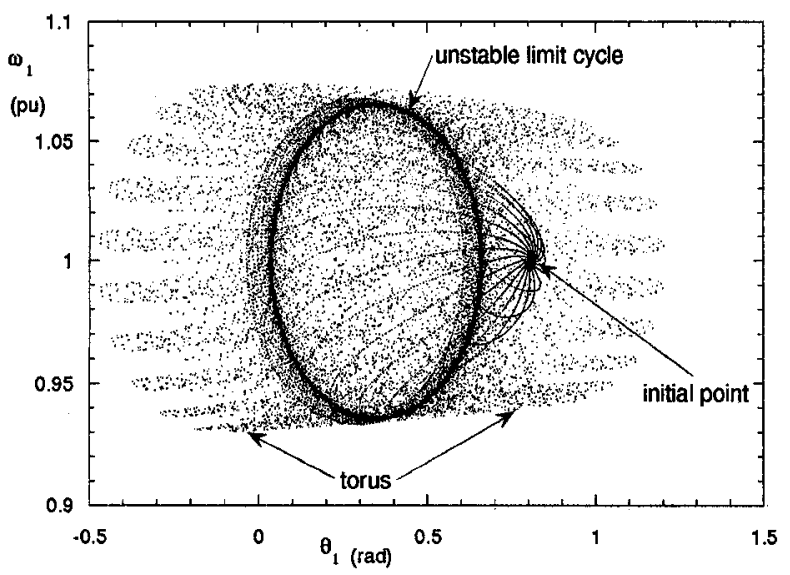

Fig. 7. Torus orbit at $X_{c}=1.2625$.

bifurcation and before the cyclic fold there are bifurcations to a stable (invariant) torus at $X_{c}=1.2621$ and $X_{c}=1.3599$. In Figs. 7 and 8 torus orbits at $X_{c}=1.2625$ and $X_{c}=1.344$ are shown in $\theta_{1}-\omega_{1}$ space, respectively.

\section{E. Dependence of System Parameters}

Among system parameters damper windings have almost same speed in response with the torsional mode. To verify some dependence of system parameters on the periodic orbits models with only one damper winding (a 1-q damper model and a 1-d damper model) were provided. Figs. 9 and 10 show the respective bifurcation diagrams. Moreover, the value of damping coefficient $D_{12}$, which has direct relation to the stability of torsional mode, has been varied. Figure 11 shows the corresponding results. Models with at least one damper winding in $q$-axis produce a branch of periodic orbits which connect the two Hopf bifurcation points. This periodic orbit surrounds the branch of unstable equilibrium points due to the SSR. Also, the damping coefficient $D_{12}$ plays an important role to de-

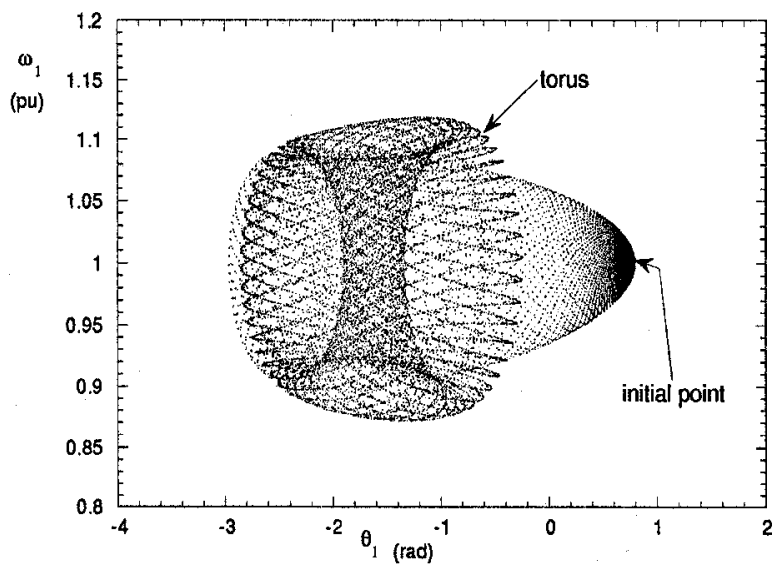

Fig. 8. Torus orbit at $X_{c}=1.344$.

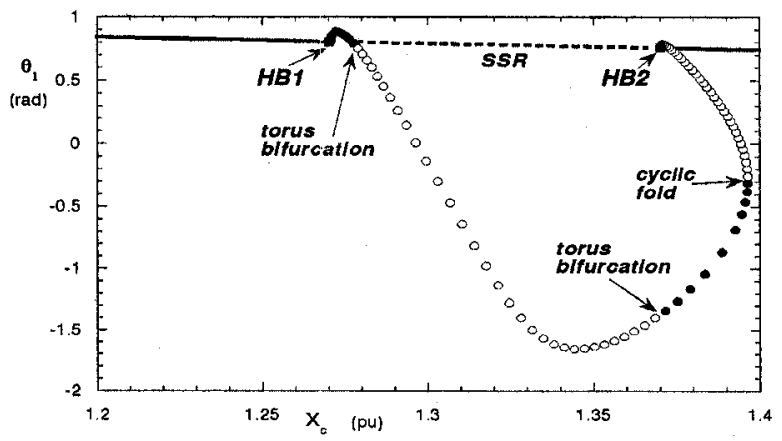

Fig. 9. Bifurcation diagram of 1-q damper model.

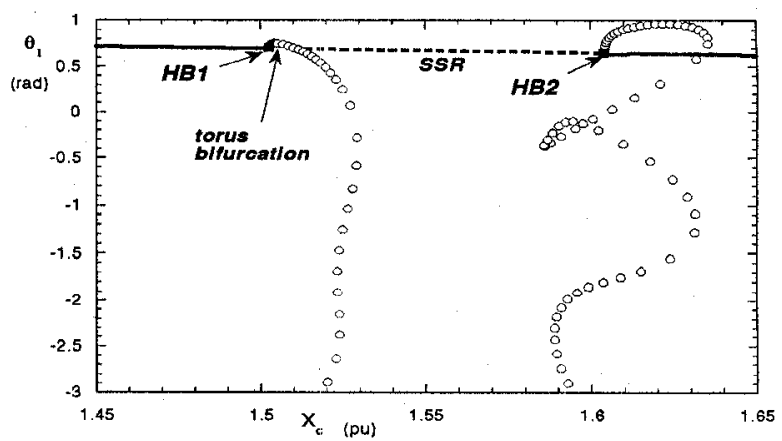

Fig. 10. Bifurcation diagram of 1-d damper model. 


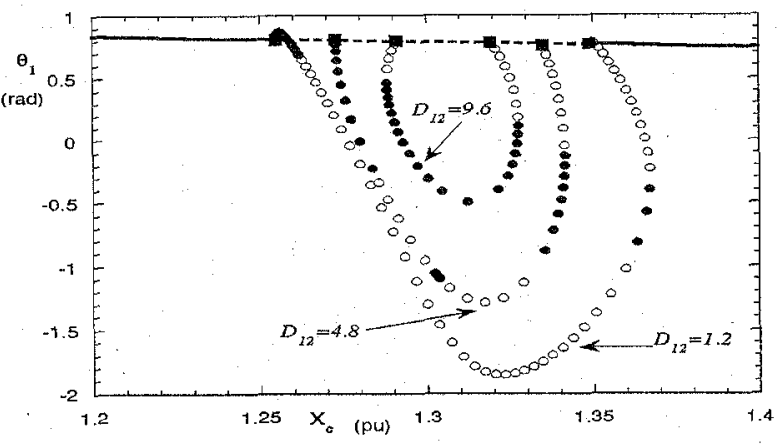

Fig. 11. Bifurcation diagram for different values of $D_{12}$.

termine the characteristics of the periodic orbits. A large value of $D_{12}$ makes the periodic orbits attractive when the model has at least one damper winding in q-axis. Note that completely same results are obtained for the model $B$ (the IEEE first benchmark model).

\section{CONCLUSIONS}

In this paper some strange nonlinear attractors appearing in the system with SSR phenomena has been described. In the bifurcation diagram there is a branch corresponding to periodic orbits which connects the two Hopf bifurcation points. In this branch, a torus bifurcation as well as cyclic fold have been detected. It has been observed that a damper winding in the $\mathrm{q}$-axis produces this sequence of bifurcations. The damping coefficient in the turbine-rotor shaft system makes the periodic orbits attractive.

\section{REFERENCES}

[1] P. M. Anderson, A.A. Fouad. Power system control and stability, Rev, print. Piscataway, NJ : IEEE Press, [1993], c1994.

[2] P. M. Anderson, B.L. Agrawal, J.E. Van Ness, Subsynchronous resonance in power systems, New York : IEEE Press, c1990.

[3] Y. Yu, Electric Power System Dynamics, London Academic Press, Inc., 1983.

[4] N. G. Hingorani A new scheme for subsynchronous resonance damping of torsional oscillations and transient torque. I. IEEE Transactions on Power Apparatus and Systems, April 1981, vol.PAS-100, (no.4):1852-5.

[5] R.A.Hedin, K.B. Stump, N.G. Hingorani, A new scheme for subsynchronous resonance damping of torsional oscillations and transient torque. II. Performance. IEEE Transactions on Power Apparatus and Systems, April 1981, vol.PAS-100, (no.4):1856-63.

[6] M.R. Iravani, A. Semlyen, Hopf bifurcations in torsional dynamics (turbine-generators). IEEE Transactions on Power Systems, Feb. 1992, vol.7, (no.1):28-36.

[7] W. Zhu, R.R. Mohler, R. Spee, W.A. Mittelstadt and D. Maratukulam. Hopf bifurcations in a SMIB Power System with SSR: IEEE PES Summer Meeting in Portland, 95 SM 531-4 PWRS, Jul, 1995.

[8] E. J. Doedel, X. Wang, T. Fairgrieve. AUTO94: Software for continuation and bifurcation problems, Applied Mathematics Report, California Institute of Technology, November 1994.
[9] E. H. Abed and P.P. Varaiya, Nonlinear oscillations in power systems, International Journal of Electric Power and Energy Systems, January 1984, 337-43.

[10] J. Guckenheimer and P. Holmes, Nonlinear Oscillations, Dynamical Systems, and Bifurcations of Vector Fields, SpringerVerlag, New York, fourth printing, 1993.

[11] E. H. Abed and N. Tsolas and P.P. Varaiya, Study of nonlinear oscillations due to exciter control, Proceedings 1983 IEEE International Symposium on Circuits and Systems, 1983, May, 1410-1413.

[12] J.C. Alexander, Oscillatory solutions of a model system of nonlinear swing equations, International Journal of Electric Power and Energy Systems, July 1986, 130-136.

[13] H.G. Kwatny and G.E. Piper, Frequency domain analysis of Hopf bifurcations in electric power networks, CAS-37, 10, October 1990, 1317-1321.

[14] C. Rajagopalan and P.W. Sauer and M.A. Pai, Analysis of voltage control systems exhibiting Hopf bifurcation, Proceedings of the 28 IEEE Conference on Decision and Control, 1989, December, 332-335.

[15] J. E. Marsden and M. McCracken, The Hopf bifurcation and its applications, Springer-Verlag, New York, 1976.

[16] Wolf, D.M.; Varghese, M.; Sanders, S.R. Bifurcation of power electronic circuits. Journal of the Franklin Institute, Nov. 1994, vol.331B, (no.6):957-99.

[17] A. A. Andronov and C.E. Chaikin, Theory of Oscillations, Princeton University Press, 1949.

[18] E. H. Abed, H.O. Wang, J.C. Alexander, A.M.A. Hamdan and H.-C. Lee, Dynamic bifurcations in a power system model exhibiting voltage collapse, Int'l Journal of Bifurcation and Chaos, Vol. 3, No. 5, 1993, 1169-1176.

[19] IEEE Committee Report, First Benchmark Model for Computer Simulation of Subshynchronous Resonance, IEEE Trans. on Power Apparatus and Systems, Vol.PAS-96, No.5, Sept. 1977, pp.1565-1572.

Yasunori Mitani is Associate Professor of Electrical Engineering at Osaka University, Japan. He obtained the Dr. of Engineering degree from Osaka University in 1986. He was a Visting Research Associate at the University of California, Berkeley from 1994 to 1995. His research interests are in the area of analysis and control of power systems.

Kiichiro Tsuji is Professor of Electrical Engineering at Osaka University. He obtained the Ph.D. degree in system engineering from Case Western Reserve University, Cleveland, $\mathrm{OH}$, in 1973. His research interests are in the areas of analysis, planning and evaluation of energy systems including electrical power system.

Matthew Varghese is an engineer at Landis \& Gyr Utilities Services Inc., San Jose. He obtained the Ph.D in Electrical Engineering from Cornell University where he was Mathematical Sciences Institute Graduate Fellow. He has taught at Clarkson University, New York and at Harvey Mudd College, Claremont. He was Assistant Research Engineer with the Electronics Research Laboratory at the University of California, Berkeley. He has been with Landis \& Gyr Utilities Services Inc. since 1996.

Felix $\mathbf{W u}$ is Professor of Electrical Engineering and Computer Sciences at the University of California, Berkeley. Ife was Tokyo Electric Power Company Chair in 1991. He is a Fellow of IEEE and is serving as the Chairman of the Fellows Working Group of the Power System Engineering Committee. He has served on the Editorial Board of the IEEE Proceedings.

Pravin Varaiya is James Fife Professor of Electrical Engineering and Computer Sciences at the University of California, Berkeley, and Director of PATH, a multi-university program in Transportation Systems. From 1975 to 1992 he also was Professor of Economics at Berkeley. Dr. Varaiya has held a Guggenheim Fellowship and a Miller Research Professorship. He is a Fellow of IEEE. 